Rev. Chll. Pediatr. 65 (1); 1-6, 1994

\title{
Perfil plasmidial de Klebsiella pneumoniae, cepas endémicas y aisladas de brotes en una unidad de neonatología
}

\author{
Leonardo Maggi C. ${ }^{1}$; Emesto Payá G. ${ }^{2}$; Patricio Nercelles M. \\ Alvaro Cordovez P. ${ }^{3}$; Juan Martínez D.6; Valeria Prado J.'; \\ Freddy Squella B. ${ }^{4}$; Carmen Mendoza N.5
}

\section{Plasmid profile of nosocomial Klebsiella pneumoniae strains}

\begin{abstract}
Plasmid profile anolysis is usefull in the differentiation of fenolipically similar baclerial strains. Our aim was to evaluole the usefuiness of plosmid profile analysis in the identilicallon of different $K$. pneumonice strains isolated from endemic and $\alpha$ ossional nosocomia infections. Seventeen $K$. oneumonige strains isolated from two separate aulbreaks fyears 1990 and 1992, twelve and tive strains respectively) at a neonatal unil [Carlos Van Buren Hospital, Valparaiso, Chile] and 31 endernk strains obtoined along year 1992 from nine different clinical wards within the same hospital were analyzed. Strains were tesled for plasmid profile analysis by gel electrophoresis and for onlimicroblal susceplibility palterns by the Kirby Bouer method. Plasmid protiles were identical for 11 of the 12 strains in outbreak 1 and for all five strains isolated in oulbreak 2 . Slrains belonging to different outbreaks had different plasmid profiles. Twentytive plasmid profiles were identilied among the 31 endernic strains. Plasmid piofile analysis allowed the ideniffcalion of one different strain in outbreak I which was not identified by antimicrobial resistance paltern analysis and also of seven similar endemic slrains that showed different ontimicrobial resistonce palterns. Flasmid profile analysis is usefull in epidemiological investigations of nosocomicl infections and able to differentiate strains more effectively than the commonly used ontimicobial resistance potterns.
\end{abstract}

[Koy words: Klebsiella pneumoniae, baclesial typing, plasmids, epidemiological matkers.]

Las infecciones intrahospitalarias (IIH) constituyen un problema de salud pública debido a su mortalidad, costo y frecuencia. En Chile se notifican aproximadamente 45000 episodios de IIH por affo, con una tasa de 4,5/100 egresos, estimándose que causan unas 2500 muertes anuales'i,

Klebsiella pneumoniae ocupa el tercer lugar como agente etiológico global, sólo superado por Staphylococcus aureus y Escherichia coli ${ }^{2}$. Produce infecciones graves de la vfa urinaria, del

1. Departamento de Microbiologia, Facultad de Medicina, Universidad de Chile.

2. Hospital Van Buren, Vajparaiso.

3. Hospital Barros Luco-Trudeau, Santiago.

4. Ayudante alumno Facultad de Medicina, Universidad de Chile.

5. Hospital Dr. Exequiel Gonźlez Cortés.

6. Tecnólogo Médico. Departamento de Microbiología, Facultad de Medicina, Universidad de Chile.

Financiamiento Proyecto Fondecyt 92-1094. aparato respiratorio y el torrente sanguíneo ${ }^{3.4}$, en forma endémica y también en brotes epidémicos de gran letalidads, especialmente si su localización es la circulación'. Además suele presentar resistencia a los antimicrobianos más comunes, lo que hace su tratamiento difícil y $\cos t 00^{7-9}$. Las unidades de neonatología son frecuentemente afectadas por epidemias de fatales consecuencias con este agente ${ }^{10-12}$. Para controlarlas es necesario disponer de métodos microbiológicos eficaces en detectar los brotes, discriminar entre cepas epidémicas y ocasionales y trazar la forma en que ocurre su diseminación entre los distintos servicios clínicos del hospital. Los marcadores epidemiológicos habitualmente utilizados con este fin son el antibiotipo, el biotipo y en algunos casos el serotipo o el fagotipo ${ }^{13}$.

Las técnicas de análisis genético (genotipificación) permiten tipificar con alto grado de discriminación cepas bacterianas con características fenotipicas similares ${ }^{14}$. El método más utili- 
zado es el análisis del ADN ubicado fuera del cromosoma de la bacteria en forma de cadenas superenrolladas, denominadas plasmidios, que pueden ser extraidos y posteriormente examinados mediante electroforesis en gel de agarosa. El estudio se puede complementar cortando los plasmidios mediante enzimas específicas (endonucleasas) y haciendo análisis del ADN cromosómico de la bacteria.

Esta investigación se realizó con el propósito de evaluar el perfil plasmidial (genotipo) como técnica de tipificación de Klebsiella pneumoniae en epidemias intrahospitalarias en recién nacidos, comparándolo con el perfil de resistencia a los antimicrobianos (antibiotipo).

\section{Materiales y Métodos}

Origen de las cepas. Se incluyeron en este estudio 52 cepas de $\boldsymbol{K}$. presunoniae aisladas de 52 pacientes internados en el hospital Carlos van Buren, de Valparaíso, Chile; un hospital general de alta complejidad que cuenta con 756 camas, que mantiene un sistema de vigilancia activa de infecciones intrahospitalarias (IIH) desde 1986. Todas ellas provenían de muestras clínicas de enfermos con diag. nóstico de infección intrahospitalaria ${ }^{1 *}$. Los ejemplares fueron clasificadas en: grupo 1 (cepas aisladas darante un brote producido en la unidad de neonatología entre mayo y noviembre de 1990; $n=12$ ); grupo 2 (cepas gisladas đurante un segundo brote producido en la misma unidad el mes de abril de $1992 ; n=5$ ); grupo 3 (cepas de pacientes de otros servicios del hospital aisladas entre abril y julio de 1992; $\mathrm{n}=35$ ).

Identificación de las cepas. Las cepas fucron identificadas como $K$. pnewmoniae siguiendo las nomas de jdentificación de enterobacterias del Institoto de Salnd Pública de Chile ${ }^{15}$

Determinación del perfil de reslstencia a los antimlcrobianos. La suceptibilidad de las cepas fue determinad a por el mélodo de difusión por discos en agar MuellerHinton (Kirby-Bauer) según las indicaciones del National Committee for Clinical Laboralory Standars (NCCLS) ${ }^{16}$. Los antimicrobianos incluidos fueron: genlamicina, cotrimoxazol, cefazolina, cefotaxima, cefoperazona, ceftriaxona, ceflazidima, amikacina, sulbactarn/ampicilina, ciprofloxacino y enoxacino. Se utilizaron como puntos de corte para determinar resistencis los establecidos por el NCCLS ${ }^{16}$; se incluyeron como control las cepas ATCC Pseudomonas aeruginaso 27853 y $E$. coli 25922.

Determinacion del perfil plasinldial. Luego de su aislamiento las cepas fueron enviadas en medio "Dorsel$\mathrm{egg}^{117}$ al centro de referencia, donde se mantuvieron a $4^{\circ} \mathrm{C} y$ a $-70^{\circ} \mathrm{C}$ en caldo de soya tripticasa (Tripticase Soy Broth) con glicerol 15\%. El ADN plasmidial fue extraído mediante una modificación de la técnica de lisis alcalina de Birboim y Doly ${ }^{18}$ como sigue: las cepas fueron inoculadas en $5 \mathrm{ml}$ de caldo Luria e incubadas a $37^{\circ} \mathrm{C}$ en agitación por 18 horas; $1,5 \mathrm{ml}$ de cultivo fueron traspasados a tutos de
Eppendorf y centrifugados a 16000 pm por $45 \mathrm{sec}$ (microcentrífuga Beckman E., Beckman Instruments, inc. P.A. Ca. EUA). E precipilado fue resuspendido en $100 \mu 1$ de solución de lisis (tisozima $2 \mathrm{mg} / \mathrm{ml}, 10 \mathrm{mM}$ EDTA, $50 \mathrm{mM}$ glucosa y $25 \mathrm{mM}$ Tris $\mathrm{Cl}(\mathrm{pH} \mathrm{B}, 0)$ e incubado a $4^{\circ} \mathrm{C}$ por 20 min; se agregaron $200 \mu l$ de solución de denaturación $(0,2$ $\mathrm{N} \mathrm{NaOH}, 1 \%$ dodecilsulfato de sodio (SDS1) y se incubú en hielo por $5 \mathrm{~min}$; posteriormente se agregó la solución de

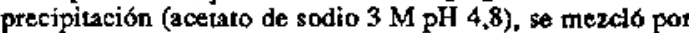
inversión 5 veces y se incubó en hielo por $15 \mathrm{~min}$; se centrifug $\delta$ a $16000 \mathrm{rmm}$ durante $15 \mathrm{~min}$ y se traspasaron $400 \mu$ del sobrengdante a tubos de Eppendorf agregandose I ml de etanol al $99,8 \%$ frío $\left(-20^{\circ} \mathrm{C}\right)$; se mezclo por inver. sión y se incubo a $-70^{\circ} \mathrm{C}$ por $15 \mathrm{~min}$.

Se centrifug 6 en microcentrífuga por $10 \mathrm{~min}$, se eliminó el sobrenadante por inversión y aspiracion suave para posteriormente agregar $200 \mu l$ de etanol $\left(99,8 \%,-20^{\circ} \mathrm{C}\right)$ y $100 \mu \mathrm{l}$ de solución contenjendo NaOAc $0,1 \mathrm{M}$ y Tris Cl 50 $\mathrm{mM} \mathrm{pH} \mathrm{8.0.} \mathrm{Se} \mathrm{incubó} \mathrm{a}-70^{\circ} \mathrm{C}$ por $15 \mathrm{~min}$, se centrifug $\delta$ a $16000 \mathrm{rmm}$, se eliunin $\delta$ el sobrenadante y se se $\varnothing \delta$ el precipitado en bornba de vacío.

Electroforesis en gei de agarosa. Se prepararon geles de agarosa $0,7 \%$ en tampón de electroforesis Tris borato IX; el ADN fue resuspendido en $16 \mu$ de agua bidestilada agregándose $4 \mu \mathrm{l}$ de solución de carga $(0,07 \%$ azul de bromofenol, 7\% SDS, 20\% Ficoll, en agus); la electrofore sis se hizo a 4 V/con durante 3 horas; terminada esta, el gel fue teñido con $150 \mu$ de solución de bromuro de etilo $(10 \mathrm{mg})$ $\mathrm{m})$ ), examinado en transiluminador de luz uliravioleta (UVP, Inc. Ca. EUA) y fotografiado (Polaroid in stant pack film 667). Como marcadores de peso molecular se usaron plasmidios de la cepa $E$. coli VS171 ${ }^{19}$, los que fueron extraidos mediante el procedirniento arriba descrito.

Analisis de los resultados. La capacidad de tipificacion de ambos métodos fue determinada por el porcentaje de cepas tipificadas y la capacidad de discriminación mediante el índice de Simpson 20 .

\section{Resultados}

Distribución de las cepas. En la tabla 1 se observa la distribución de las cepas por servicio y tipo de muestra. Los grupos 1 y 2 corresponden a los brotes detectados en el servicio de neonatología en 1990 y 1992 respectivamente, la mayor parte $(10 / 17)$ de las cepas provenían de hemocultivos. En el grupo 3 se incluyeron cepas aisladas de distintos servicios, durante los meses de abril a junio de 1992, destacando la mayor frecuencia en neurología y urología (33\%), siendo el urocultivo la muestra que aportó la mayor proporción (40\%).

Determinación del antibiotipo. En las tablas 2, 3 y 4 se detallan los perfiles de resistencia antimicrobiana y los plasmidiales de las cepas de los grupos 1, 2, y 3 respectivamente. Encontramos 25 patrones de resistencia a los 11 antimicrobianos probados; en 14/52 cepas de $K$. pneu- 
Tabla 1

Distribución de cepas por servicio clínico

\begin{tabular}{lcr}
\hline Servicio clínico & n de cepns & $\%$ \\
\hline Neonatología & 17 & 32,7 \\
Urologia & 9 & 17,3 \\
Neurocirugla & 8 & 15,4 \\
Medicina & 4 & 7,7 \\
UCI & 3 & 5,7 \\
Cingéa infantil & 3 & 5,7 \\
UCI pediátrica & 3 & 5,7 \\
Traumatología & 2 & 3,8 \\
Pediatría & 2 & 3,8 \\
Ginecología & 1 & 1,9 \\
\hline Total & 52 & 100,0 \\
\hline
\end{tabular}

Tabla 2

Frecuencia de aislamiento según muestra de cepas del grupo 3

\begin{tabular}{lrr}
\hline Muestra & n & \% \\
\hline Orina & 21 & 40,3 \\
Secreción bronquial & 6 & 11,5 \\
Herida operatoria & 3 & 5,7 \\
Secreción traqueal & 2 & 3,8 \\
Secreción otica & 1 & 1,9 \\
Sangre & 1 & 1,9 \\
Abceso cerebral & 1 & 1,9 \\
\hline Total & 35 & 67,0
\end{tabular}

Tabla 3

Perfiles de resistencis

\begin{tabular}{llc}
\hline Tipo & Reslstencle & Frecuencia \\
\hline 1 & G. Co, Cef, Ctx, Cfp, Ctr, Ak, Sam & 14 \\
2 & G. Co, Cef, Cfp, Sam & 5 \\
3 & G, Co, Sam & 3 \\
4 & G, Co, Cef, Crx, Cfp, Ctr, Caz, Ak, Sam & 2 \\
5 & G, Co, Cfp, Ctr, Sam & 2 \\
6 & Cef, Ctr, Sam & 2 \\
7 & Cef, Ctr & 2 \\
8 & G, Co, Ctr, Sam & 2 \\
9 & Co, Cfp & 2 \\
10 & Co, Cfp. Sam & 2 \\
11 & G, Co, Cfp, Sam & 2 \\
$12-25$ & (14 antibiotipos distintos) & 14 \\
\hline Total & & 52 \\
\hline
\end{tabular}

G: gentamicina; Co: cotrinoxazol; Cef: cefazolina; Ctr: ceftriaxons; Cfp: cefoperazona; Cox: cefotaxima; Caz: Cefta. zidina; Ak: Amikacina; Sarn: sulbactam/ampicilina; Cip: ciprofloxacino, En: enoxacino. moniae $(27 \%)$ el patrón de resistencia era tipo 1 y correspondieron a todas las cepas del grupo $1(12 / 12)$ y a dos cepas del grupo $3(2 / 35)$. $5 / 52$ cepas presentaron patron tipo $2(9,6 \%)$ y correspondieron a la totalidad de las cepas del grupo 2 (5/5). Las restantes 33 cepas presentaron patrones diversos y correspondieron a cepas del grupo 3, en el cual cuatro cepas eran resistentes simultáneamente a cefotaxima y ceftazidima, tres de ellas eran, además, resistentes a amikacina.

Determinación del perfíl plasmidial. En las tablas 2,3 y 4 se detallan los perfiles plasmidiales de las cepas de los grupos 1,2 y 3 respectivamente. Este se realizo en 48 de las 52 cepas; 4 cepas no fueron recuperables $(7,7 \%)$. Encontramos 25 perfiles plasmidiales en 46 cepas. Dos cepas no fueron tipificables. El perfil tipo A se presentó exclusivamente en las del primer brote (gnupo 1), donde 11 de 12 tenían dicho perfil, mientras otra presentó un perfil similar pero con una banda agregada de $10 \mathrm{Md}$ (perfil tipo E). El perfil tipo B se encontró en siete cepas del grupo 3 , las que tenían antibiotipos distintos.

El perfil tipo $\mathrm{C}$ fue exclusivo de las cepas del segundo brote (grupo 2) y se encontró en todas ellas (5/5). El perfil tipo D se encontró en 2 cepas del grupo 3 y sus antibiotipos resultaron distintos. Los perfiles tipos $\mathrm{F}$ al $\mathrm{Y}$ se encontraron individualmente en 20 cepas del grupo 3. Las cepas multirresistentes presentaron patrones plasmidiales diferentes.

Eficacia de la tipificación. La capacidad de tipificación fue de $100 \%$ para el biotipo y de 95,83\% para el perfil plasmidial. El indice de discriminación fue 0,915 para el antibiotipo y 0,922 para el perfil plasmidial.

Tabla 4

Perfiles plasmidiales

\begin{tabular}{llc}
\hline Tipo & PJasmidios (md) & Frecuencla \\
\hline 1 & 7,$5 ; 6,0 ; 3,8$ & 11 \\
2 & 52,0 & 7 \\
3 & 25,$2 ; 21,0$ & 5 \\
4 & 70,$0 ; 52,0 ; 7,5 ; 4,3 ; 3,4 ; 1,2 ; 1,0$ & 2 \\
5 & $10.0,7.5,6.0,3.8$ & 1 \\
$6-25$ & $(20$ perfiles distinos) & 20 \\
\hline Total & & 46 \\
\hline
\end{tabular}




\section{Comentario}

Las infecciones intrahospitalarias constituyen un creciente problema, en nuestro país y en el mundo ${ }^{1,21}$. El apoyo del laboratorio de microbiología es fundamental para orientar el estudio epidemiológico, especialmente antc la aparición de brotes por patógenos que por su frecuencia, multiresistencia y letalidad constituyen verdaderos desafíos para los equipos encargados del control de las IIH.

$K$. pneumoniae cumple con condiciones que lo hacen ser un patogeno nosocomial por excelencia, ya que produce infecciones intrahospitalarias en pacientes adultos y pediátricos en hospitales de alta y baja complejidad. Es un agente frecuente en las unidades de recién nacidos, donde produce graves brotes epidémicos y probablemente enterocolitis necrótica ${ }^{22}$. El reservorio principal parece ser el intestino de los pacientes ${ }^{23}$ y se ha propuesto el mecanismo de traslocación bacteriana para explicar su diseminación desde el tubo digestivo a la sangre ${ }^{24}$.

Por esa razón, en algunos centros se hace rutinariamente vigilancia de $K$. pneumoniae mediante cultivos de deposición de pacientes en ratamiento intensivo y recién nacidos, para detectar sobrecrecimiento y colonización por cepas multirresistentes ${ }^{25}$. Algunos han propuesto, entre las medidas de control, profilaxis con antibióticos en pacientes seleccionados ${ }^{23}$. Sin embargo, en nuestra experiencia to que ha permitido el control de los brotes han sido la supervisión y el cumplimiento estricto de las normas para procedimientos invasivos y no nos parece útil el cultivo habitual ni el uso de profilaxis en pacientes colonizados.

A partir de $1983 K$, pneumoniae adquiere una nueva dimensión como patógeno multirresistente, con la aparición -en Alemania- de cepas productoras de beta lactamasas de espectro expandi$\mathrm{do}^{26}$; desde entonces se han comunicado numerosos brotes por estas $\operatorname{cepas}^{27.28}$, produciendo preocupación la capacidad de transferencia de determinantes de resistencia entre cepas de $K$. pneumoniae y otras enterobacterias mediante la diseminación de plasmidios, fenómeno que se produce en forma frecuente y rápida ${ }^{29}$.

En el Hospital Carlos van Buren, de Valparaiso, se ha organizado un sistema de vigilancia selectiva de infecciones hospitalarias, que ha permitido identificar problemas en forma más espe- cífica; sin embargo es necesario contar con técnicas microbiológicas discriminativas, especialmente en presencia de un brote, ya que la eficiencia de las medidas para prevenir la diseminación de paciente a paciente, de cepas epidémicas, dependerá de la capacidad para identificar los reservorios y mecanismos de trasmisión.

Uno de los problemas aún no resueltos en la investigación de brotes de infección hospitalaria se refiere a los marcadores epidemiologicos útiles para identificar las cepas involucradas. Se han propuesto muchos métodos de tipificación para distintos patógenos; en el caso de $K$. pneumoniae varios pueden emplearse por su costo o su complejidad técnica. La serotipificación basada en el antígeno capsular no está disponible en el comercio, mientras reproducibilidad y sensibilidad de técnicas como biotipificación, tipificación por bacteriocinas y por fagos son bajas ${ }^{30}$. Por esta razón, en nuestro hospital el marcador habitualmente utilizado es el perfil de resistencia o antibiotipo.

Las tếcnicas de epidemiología molecular se han utilizado para trazar la diseminación de casi todos los patógenos de importancia nosocomial, evitan la influencia de factores ambientales que alteran el fenotipo, son relativamente rápidas y pueden ser aplicadas sin mayores variaciones al estudio de distintas especies bacterianas.

En el presente estudio evaluamos la eficiencia del antibiotipo y el perfil plasmidial como métodos de tipificación de cepas de $K$. pneumoniae aisladas de infecciones intrahospitalarias. Si tomamos como marcador exclusivamente el antibiotipo, el primer brote fue producido por una cepa única y multirresistente, sin embargo el análisis genético reveló la existencia de otra cepa con características fenotípicas similares pero diferente contenido plasmidial, lo que sugiere la presencia de dos clones, uno de tipo epidémico y otro como infección aislada; esto concuerda con lo encontrado por otros autores, en el sentido que el análisis del material genómico permite discriminar cepas fenotípicamente similares pero no relacionadas genéticamente ${ }^{25}$. Es interesante señalar que mientras más marcadores se utilizan, más clones es posible detectar, por lo que algunos recomiendan emplear varios, especialmente cuando uno de ellos es el análisis plasmidial, pues se han descrito cepas con idéntico perfil que, por estudios más finos del genoma, fueron 
asignadas a clones diferentes, usando como patrón de referencia el análisis del polimorfismo de los fragmentos de restricción de $\mathrm{ADN}$ que codifican el ARN ribosomal (ribotipificación) ${ }^{25}$. Sin embargo, el análisis plasmidial parece ser un buen marcador epidemiologico, siempre que se tome en cuenta esta limitación.

Las cepas resistentes a ceftazidima, ceftriaxona y amikacina, posiblemente corresponden a especímenes aislados, no relacionados genéticamente, a pesar que en dos de ellas los antibiotipos inducen a pensar en infección cruzada. Se ha establecido que la diversidad genetica de las cepas multirresistentes es amplia y se han descrito brotes por cepas multiresistentes, con antibiotipos similares, que correspondian a 15 ribotipos diferentes $^{25}$. La mejor correlación entre antibiotipo y perfil plasmidial se encontró en el segundo brote, lo que sugiere que éste fue causado por un clon único, siendo ambas técnicas útiles en este caso. Los análisis genético y fenotípico mostraron gran heterogeneidad en las cepas no relacionadas con brotes, lo que apoya la tesis de que en el caso de endemia, la infección es probablemente endogena y no cruzada.

Dos hechos llaman la atención en el análisis de las cepas del grupo 3; el primero es que encontramos ocho antibiotipos distintos, con dos cepas cada uno, y un antibiotipo con tres cepas, lo que podría sugerir en un primer momento la existencia de infección cruzada, sin embargo los perfiles plasmidiales fueron diferentes, e indican que probablemente se trata de infecciones aisladas. Por otro lado, basados en el perfil plasmidial, hubo probablemente un brote que afectó los servicios de medicina, neurocirugia, urología y cuidados intensivos de pediatría, involucró a siete pacientes y no pudo ser detectado por el antibiotipo.

Las cepas que presentaron el perfil tipo D, probablemente están relacionadas genéticamente y también sería conveniente realizar en ellas estudios más finos, como corte de los plasmidios y análisis de A.DN cromosómico, para confirmar su pertenencia a un clon único.

La menor capacidad de tipificación del perfil plasmidial que del antibiotipo otorga al primero menor sensibilidad; el mejor índice de discriminación para el perfil plasmidial implica que la probabilidad de asignar correctamente una cepa a un tipo deterninado es mayor cuando se utiliza este que el antibiotipo.
Estos resultados indican que las cepas endémicas de los distintos servicios del hospital son genéticamente heterogéneas, lo que resulto concordante con los antibiotipos encontrados. Las cepas provenientes de los brotes en neonatología resultaron homogeneas en sus perfiles plasmidiales y antibiotipos, excepto una cepa del primer brote; esto sugiere que ambos fueron producidos por sendos clones epidémicos. El antibiotipo es un mancador adecuado en el estudio de infecciones intrahospitalarias por $K$. pneumoniae; sin embargo, en el caso del estudio especifico de un brote por este agente, el perfil plasmidial prestaría mayor utilidad ya que permite identificar mejor los clones involucrados y discrimina satisfactoriamente cepas epidémicas y endémicas. Esto pennite identificar la vfa de transmisión al estudiar reservorios y adoptar la intervención adecuada.

Por último, se debe destacar la importancia del estudio de las bases genéticas de la resistencia a antimicrobianos en las cepas de origen intrahospitalario, ya que así como se diseminan cepas multirresistentes, se pueden diseminar plasmidios entre cepas de distintas especies. $K$. pneumoniae ha sido identificado como el principal reservorio de plasmidios $\mathbf{R}$ capaces de transferirse entre especies, lo que asigna un segundo objetivo de importancia a la determinación del perfil plasmidial.

\section{Resumen}

El perfil plasmidial es utilizado frecuentemente en estudios epidemiológicos para distinguir cepas con características fenotípicas similares. Para evaluar la utilidad de esta lécnica en la tipificación de $K$. pneumoniae, estudiamos dos brotes de infección intrahospitalaria ocurridos en la unidad de neonatología del Hospital Carlos van Buren. Se analizó el perfil plasmidial de 12 cepas aisladas de un brote producido en 1990,5 cepas aisladas de un brote ocurrido en 1992 y 31 cepas aisladas durante 1992 en otros servicios del hospital y no relacionadas con brotes. Como patron fenotípico se utilizó el perfil de resistencia a los antimicrobianos (antibiotipo). Del primer brote 11/12 cepas presentaron el mismo perfil plasmidial y $5 / 5$ cepas del segundo brote, siendo ambos perfiles distintos entre sí. Los patrones plasmidiales de las cepas endémicas de otros 
servicios fueron variables y diferentes de las cepas epidémicas. En ambos brotes, el antibiotipo de las cepas epidémicas fue concordante con el patrón plasmidial, excepto en una cepa del primer brote. Estos resultados sugieren que el análisis del perfil plasmidial es una técnica adecuada para discriminar cepas epidénicas y endémicas de $K$. pneumoniae y por lo tanto puede ser útil en estudios de brotes por este agente.

(Palabras clave: Klebsiella Pneumoniae, tipificación bacteriana, perfil plasmidial, infecciones hospitalarias.)

\section{Referencias}

1. Otake F, Brenner $P$ : Informe de la vigilancia epidemiologica y actividades de control de las infecciones intruhospitalarias en Chile. Ministerio de Salud, Chile. 1989.

2. Olaka F, Brenner P: Vigilancia de las infecciones intrahospilaln rias en Chile. Primer congreso chileno de epiderniologia. Gredis editores, Stgo., Chile, 1990.

3. Palomino C: Prevalencia de infección nosocomial observada en 1988 en el hospital de enfermedades infeccjosas Dr. Lucio Cóndova. Rev Chil Infect 1990; 7: 51-57.

4. Fernandez A, Pinto ME: Bacteremin intrahospitala. ria, evsluación de cinco años. Rev Chil Infect 1991; 8: 158-161.

5. Wazanakwnakorn C, Jura J: Klebsiella bacteremis: a review of 196 episodes during a decsde (1980 - 1989). Scand J Infect Dis 1991; 32: 399.405.

6. Otaiza $F, B$ renner $P$ : Infecciones intrahospitalarias del torrente sanguíneo en Chíle, cinco afios de vigilancia epidemiólogica. Rev Chil Infect 1992; 9; 48-56.

7. Pinto ME: Rexistencia de bacilos Gram negativos y Staphylococeus awews en Chile. Rev Chil Infect 1989: 6: 213-214.

8. Montiel F: Evolución de la resistencia de bacilos Gram negativos frente a cefalosporinss. Rev Chil Infect 1989; 6: 220-226.

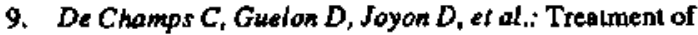
a meningitis due to an Enterobacter aerogenes producing a depressed cephalosporinase and a Kiebsiella pneumonjae producing and extended-speclrum beta-lactemase. Infection 1991; 19: 181-183

10. Tellerlas $L$, Oto $M$, Lagos $E$, ef al.: Infecciones bacterianas neonstales: magnitud y aspectos clínicos. Rev Chil Pedints 1989; 60: 262-266.

11. Grauel $E$, Halle $E$, Bollmann $R$, ef al.: Neonatal septicaemia: incidence, etiology and outcome. A six years analysis. Acta Paediatr Scand Suppl 1989; 360: 113119.

12. Guerrero P: Infeccion intrahospitalania por Klebsiella pnewnoniae en un broce de sepsis en reciton nacidos. Rev Chil Pediatr 1981; 52: 306-313.
13. Rubih $S J$ : Klebsiella markers sisteans. Infect Control $1985 ; 6: 59-63$.

14. Shlaes D, Currie-HcCumber $C$ : Plasmid analisys in molecular epidemiology: a summary and future direc. tions. Rev Inf Dis 1986; 8 : 738-746.

15. Valenzwela $M E$, Cones JA: Tablas TLM para identificacion de enterobacterins. Instituto de Salud Pública, Chile. 1991.

16. National Commillee for Clinical Laboratory Standards: Performance Siandards for Antimicnobial Disk Susceptibility Test. $4^{\star}$ ed. Villanova. Pa: National Committee For Clinical Laboratory Standards; 1988.

17. Dorser E: American Medicine $1902 ; 3: 555$.

18. Birboin $H, D$ oly $J$ : A rapid alkaline extraction procedure for screening recombinant plasmid DNA. Nucleic Acid Res 1979; 7: 1513-1523.

19. Macrina FL, Kopecko DJ, Jones KR er al.: A multiple plasmid-contajning Escherichio coli strain. Convenient source of size reference for plasmid molecules. 1978; 1: 417-420.

20. Huster PR, Gaston MA: Numericsl index of the discriminatory ability of typing systems: An application of Simpson's index of diversity. J Clin Microbiol 1988; 26: 2454-2466.

21. Schaberg DR, CWver DH, Gaynes RP: Major trends in the mictobial etiology of nosocomial infections. Am J Med 1991: 91(3B): 728-758.

22. Knight P, Cassady $G$ : Control of infection due to Klebsiella pnewnoniae in an intensive care nursery. J Perinatol 1990; 10: 357-360.

23. Taylor $M E$, Oppenheim BA: Selective decontamination of the intestinal tract as an infection control messure. J Hosp Infect 1991: 17: 271-278.

24. Lambert-Zechovsky $N$, Bingen $E$, Denamur E. et al. Molecular analisys provides evidence for the endoge nous origin of bacteremia and meningitis due to Enterobacter cloacae in an infant Clin Infec Dis 1992; 15:30-32.

25. Bingen E, Desjordiss $P$, Arier $G$, et al .: Moleculsr epidemiology of plasmid spread among extended broadspectrum beta-laclomase-producing Klebsiella pnev. moniae isolates in a pediatric hospital. J Clin Misrobiol 1993; 31 : 179.184.

26. Kliebe C, Niels BA, Meyer JF, et al.: Evolution of plasmid coded resistance to broad spectrum cephalosporine. Antimicrob Agents Chemother 1985; 28:302-307.

27. De Champs $C$, Rouby $D, G$ Gelon D, ef al.: A case control study of an outbreak of infections caused by Klebsiclla prewmonize stroins producing CTX-1 (TEM3) bela-lactamase. J Hosp Infect 1991; 18:5-13.

28. Arlet $G$, Samson-le-Pors $M J$, Rouvean $M$, el al,: Outbreak of nosocomial infection due to Klebsiella pneumoniae producing SHV-4 beta lactamase Eur J Clin Microbiol Infect Dis 1990, 9:797-803.

29. Philippon A, Ben.Redjeb S, Fournier G, at al.: Epidemiology of extended-spectrum betalactamases, Infection $1989 ; 17$ : 347-354.

30. Gaston MA, Ailyng-Smith BA, Pü $T L$ : New becteriophoge typing scheme for division of the frequent capsular serotyping of Klebsiella spp. J Clin Microbiol 1987; 25: 1228-1232. 